\title{
NOUVELLE
}

\section{Adaptation conjointe des racines à l'inondation et aux nutriments du sol}

\section{Rôle d'une protéine kinase de type MAP3K}

Zaigham Shahzad, Christophe Maurel

> La plupart des plantes terrestres utilisent leurs racines pour s'ancrer solidement dans le sol et en tirer l'eau et les nutriments minéraux nécessaires à leur croissance et leur développement. Du fait de l'hétérogénéité des sols et des effets du climat, les racines, bien qu'enfouies, sont soumises à des conditions très variables. La sécheresse, les inondations, des déficits en nutriments ou des attaques par des pathogènes, peuvent représenter des stress sévères pour les racines et la plante. Contrairement aux animaux, les plantes ne peuvent s'échapper rapidement face aux agressions de l'environnement. Elles doivent donc être capables de les percevoir et d'y répondre de manière rapide et efficace. Les mécanismes moléculaires et cellulaires mis en jeux par les plantes pour répondre à des stress individuels ont déjà été largement décortiqués dans les laboratoires. Toutefois, en conditions naturelles ou en champ, les plantes sont souvent confrontées à des situations plus complexes, combinant des stress environnementaux multiples. Leurs modes de réponse à ces contextes complexes sont encore mal connus.

Afin de croître continûment et absorber eau et nutriments, les jeunes racines manifestent une forte activité métabolique et respirent intensément l'oxygène $\left(\mathrm{O}_{2}\right)$ qui diffuse dans les cavités du sol. Ce processus est freiné en cas d'inondation ou d'irrigation excessive, engendrant un déficit en $\mathrm{O}_{2}$ (hypoxie) et un stress métabolique profond des cellules racinaires. Ces conditions de stress ont des répercutions importantes sur les productions agricoles mondiales $[1,2]$. Selon certaines estimations, l'Europe affiche chaque année 5 milliards d'euros de pertes de récoltes agricoles, simplement dues aux débordements des rivières. Ces pertes pourraient doubler d'ici 2080 du fait des changements climatiques [2]. La compréhension des modes de réponse des plantes aux inondations représente ainsi un enjeu agronomique majeur [3]. Comme bien d'autres organismes aérobies, les plantes survivent à l'hypoxie en reprogrammant l'expression de leur génome et leur métabolisme, afin de produire de l'énergie chimique par fermentation alcoolique $[3,4]$. On trouve chez les plantes une classe de facteurs de transcription, nommés RAP2 (related to AP [Apetala] 2), qui jouent un rôle analogue à celui des facteurs de transcription HIF-1

(hypoxia inducible

$(\rightarrow)$ Voir la Synthèse factor 1) chez les animaux $[5,6](\rightarrow)$. Les facteurs RAP2 de $\varepsilon$. Gothié et J. Pouysségur, $m / s$ $n^{\circ} 1$, janvier 2002, page 70

sont impliqués dans la réponse primaire à $l^{\prime} \mathrm{O}_{2}$, en déclenchant une réponse transcriptionnelle anaérobie [4]. En présence d'O $\mathrm{O}_{2}$ (normoxie), un résidu cystéine (Cys) conservé en position 2 des RAP2 est oxydé par une cystéine oxydase spécifique des plantes, provocant leur dégradation par une voie obéissant à la règle du $\mathrm{N}$-terminus ${ }^{\mathrm{l}}$

\footnotetext{
${ }^{1}$ La règle du $\mathrm{N}$-terminus (ou $\mathrm{N}$-end rule) spécifie que certains résidus, quand ils occupent la position $\mathrm{N}$-terminale d'un peptide, peuvent donner un signal de destruction.
}

Biochimie et physiologie moléculaire des plantes, UMR5004, INRA/CNRS/Montpellier SupAgro/Université Montpellier, 2, place Viala, F-34060 Montpellier, France. christophe.maurel@cnrs.fr

[7]. En condition d'hypoxie, par contre, les RAP2 sont stabilisés et transférés dans le noyau où ils activent des gènes de réponse à I'hypoxie (GRH) [4].

La réponse adaptative primaire des racines à l'hypoxie passe également par une diminution de l'absorption des nutriments et de la perméabilité racinaire à l'eau (conductivité hydraulique racinaire, $\left.L p_{r}\right)$ [8]. Les nutriments du sol, tels que le nitrate, le phosphate ou le sulfate, exercent des effets positifs ou négatifs sur la $L p_{r}$ en modulant l'activité de protéines canal hydrique, les aquaporines [9]. Malgré ces liens avérés entre disponibilité en eau, nutriments et $\mathrm{O}_{2}$ dans le sol, les mécanismes de signalisation permettant l'adaptation des plantes à la combinaison de stress nutritionnels et anaérobie restent inconnus.

Un travail récent [10], utilisant la plante modèle Arabidopsis thaliana, a permis d'identifier un gène nommé hydraulic conductivity of root 1 (HCRI). Comme nous le détaillons dans la suite de cette Nouvelle, HCRI réduit l'absorption racinaire d'eau, spécifiquement quand le milieu des racines est pauvre en $\mathrm{O}_{2}$ et riche en potassium $\left(\mathrm{K}^{+}\right)$. Dans ces conditions, $H C R I$ déclenche la réponse transcriptionnelle anaérobie en agissant sur la stabilité d'un RAP2 (RAP2.12). Nos résultats nous ont conduit à proposer que la variabilité naturelle d'HCRI sous-tend diverses stratégies de croissance des plantes face aux inondations [10]. 
A

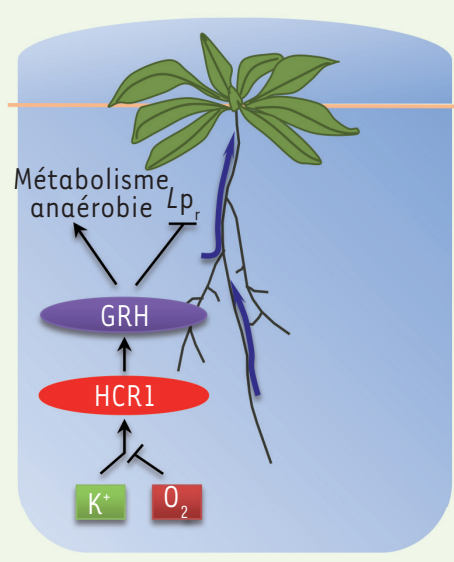

Submersion complète
B

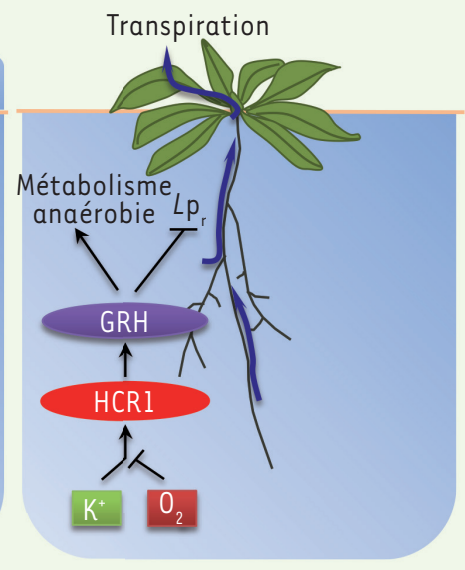

Engorgement du sol

Figure 1. Mode d'action d'HCRI dans deux contextes d'inondation. A. Submersion complète de la plante. Le déficit en $\mathrm{O}_{2}$ dans le sol, associé à la présence de $\mathrm{K}^{+}$, mène à l'activation d'une voie de signalisation impliquant HCRl (hydraulic conductivity of root 1 ). Celle-ci provoque une forte induction de gènes régulés par l'hypoxie (GRH), une activation du métabolisme anaérobie et une inhibition de la conductivité hydraulique racinaire $\left(L p_{r}\right)$. Ces réponses augmentent la tolérance de la plante à la submersion et la préparent à une réponse de croissance optimale lorsque l'inondation sera passée. B. Engorgement du sol. Dans ces conditions, la transpiration de la plante associée à une inhibition de la $L p_{\mathrm{r}}$ peut mener à un flétrissement des feuilles. Des formes alléliques d'HCRI peu ou pas actives, telles qu'on peut en trouver chez certaines accessions naturelles, pourraient présenter un avantage adaptatif en favorisant le transport racinaire d'eau. Les flèches bleues indiquent le transport d'eau dans la plante.

HCR1, un gène qui contrôle négativement l'hydraulique racinaire d'Arabidopsis

Notre but initial était d'identifier des gènes contrôlant les propriétés hydrauliques des racines de plantes. Le développement d'un système semi-automatique de mesures de la $L p_{r}$, assemblant huit chambres à pression, a permis de développer une approche de génétique quantitative de ce caractère. Des lignées recombinantes autocroisées, issues de deux accessions ${ }^{2}$ parentales naturelles d'Arabidopsis thaliana (appelées Col-0 et Bur-0), ont permis d'identifier des locus quantitatifs ( $Q T L$, quantitative trait locus) déterminant la $L p_{r}$. La carto-

\footnotetext{
${ }^{2}$ Une accession désigne un isolat unique de plante sauvage, caractérisé notamment par sa zone géographique d’origine, et consigné dans une banque de ressources.
}

graphie fine d'un de ces locus et diverses validations génétiques ont ensuite conduit à $H C R l$, un gène régulant négativement la $L p_{r}$. Nous avons observé, en particulier, que la mutagenèse par insertion d'HCRI (générant des mutants hcrl) augmente la $L p_{r}$. La protéine HCRI appartient à la famille des MAP3K (mitogen-activated protein kinase kinase kinase) de type Raf. Elle contient à son extrémité $\mathrm{N}$-terminale un domaine d'interaction protéine-protéine de type Phox(phagocytic oxidase)/Bemlp(bud emergence protein 1 ), appelé $\mathrm{PBl}$, et, à son extrémité $\mathrm{C}$-terminale, un domaine protéine kinase. Sept formes alléliques majeures d'HCRl ont pu être identifiées à partir d'accessions naturelles. Quatre de ces formes alléliques, dont celles issues des parents Col- 0 et Bur-0, manifestent des activités différentes dans un test de phosphorylation in vitro ou lorsqu'elles sont réintroduites dans le mutant $h c r l$. Par exemple, l'allèle de Bur-0 inhibe plus fortement la $L p_{r}$ que l'allèle de Col-0 dans le même fond hcrl. Par contre, un autre allèle (issu de l'accession Fei-0) semble inactif. Le rôle général des MAP3K dans la signalisation cellulaire chez les eucaryotes suggère qu'HCRl pourrait établir un lien entre signaux endogènes ou environnementaux et régulation de la $L p_{r}$. De fait, on observe que l'inhibition de la $L p_{r}$ par HCRl ne se manifeste que dans des conditions combinant hypoxie et présence de $K^{+}$. En accord avec l'idée qu'HCRl permet à la plante d'intégrer la disponibilité conjointe en $\mathrm{K}^{+}$et $\mathrm{O}_{2}$, l'ARN messager d'HCRl, ou la protéine fusionnée à une protéine rapportrice fluorescente, s'accumulent préférentiellement en présence de $\mathrm{K}^{+}$et carence en $\mathrm{O}_{2}$.

\section{HCR 1 contrôle les effets positifs du $\mathrm{K}^{+}$sur la réponse transcriptionnelle anaérobie}

Une analyse transcriptomique comparant plantes sauvages et mutantes pour horl a révélé, en présence de $\mathrm{K}^{+}$ et carence en $\mathrm{O}_{2}$, une sous-induction de nombreux GRH, dont des enzymes de la voie de fermentation (alcohol dehydrogenase 1 [ADHI], pyruvate decarboxylase $1[\mathrm{PDCl}])$. Ainsi, HCRl régule de manière positive la réponse transcriptionnelle anaérobie. En revanche, $H C R I$ ne semble pas être impliqué dans l'expression de gènes reliés à l'homéostasie du $\mathrm{K}^{+}$. On observe toutefois qu'une part significative (30 à $50 \%$ ) de l'induction des GRH est dépendante du $\mathrm{K}^{+}$, et ce de manière strictement dépendante de l'activité d'HCRI. La réponse transcriptionnelle dépendante de HCRI offre donc un parallèle frappant avec ses effets sur la $L p_{r}$. Ces deux réponses permettent d'identifier un nouveau lien entre perceptions du $\mathrm{K}^{+}$et de l' $\mathrm{O}_{2}$, lien qui est totalement sous le contrôle d'HCRI.

Bien qu'associé de près à la réponse primaire des plantes à I'hypoxie, HCRI 
n'affecte pas l'expression génique des facteurs de transcription RAP2. On observe par contre qu'en présence de $\mathrm{K}^{+}$et déficit d' $\mathrm{O}_{2}$, l'accumulation de la protéine RAP2.12 est plus faible chez les mutants horl que chez des plantes sauvages (Col-0). Par ailleurs, le domaine kinase d'HCRl est capable de phosphoryler RAP2.12 in vitro. Des plantes transgéniques sur-exprimant RAP2.12 montrent, par rapport à des plantes sauvages Col-0, un phénotype opposé à celui de plantes hcrl, c'est-à-dire une réduction de $L p_{r}$, spécifiquement en conditions hypoxiques et présence de $\mathrm{K}^{+}$. L'intégration par $\mathrm{HCRl}$ des teneurs externes en $\mathrm{O}_{2}$ et $\mathrm{K}^{+}$, en augmentant l'abondance des facteurs RAP2, provoque ainsi une activation transcriptionnelle des GRH et une inhibition du transport racinaire d'eau $\left(L p_{r}\right)$ (Figure I).

\section{HCRI contrôle la croissance de la plante en conditions d'inondation} Que signifie la perception conjointe par la plante des teneurs en $\mathrm{O}_{2}$ et $\mathrm{K}^{+}$dans le sol ? Alors que le déficit en $\mathrm{O}_{2}$ est un signe direct de l'inondation, le $\mathrm{K}^{+}$ pourrait être un indicateur des possibilités de croissance de la plante, en relation avec un lessivage éventuel du sol. Le $\mathrm{K}^{+}$contribue en effet, en tant que cation intracellulaire majoritaire, à l'entrée osmotique d'eau dans les cellules et à leur pression de turgescence ${ }^{3}$, moteur de la croissance. Selon leur intensité, les inondations provoquent un simple engorgement du sol ou submergent totalement la végétation. Ces deux scénarios ont un impact

\footnotetext{
${ }^{3}$ La résistance mécanique de la paroi cellulaire limite l'entrée osmotique d'eau dans la cellule, générant ainsi une pression hydrostatique intracellulaire dite pression de turgescence. Le relâchement de la paroi permet l'expansion des cellules et la croissance des organes de la plante.
}

différent sur le statut hydrique et la croissance des plantes [3], impliquant des facettes distinctes de la fonction d'HCRl (Figure 1). Lorsque la plante est totalement submergée, elle arrête de croître. L'activation d'HCRl favorise néanmoins sa réponse métabolique anaérobie et lui donne un fort avantage de croissance, en présence de $\mathrm{K}^{+}$, lors de la phase de récupération après inondation. Lorsque seules les racines sont inondées, la plante manifeste, de manière paradoxale, un flétrissement des feuilles car elle continue à transpirer alors que la $L p_{r}$ est réduite. Des génotypes ayant des allèles inactifs ou peu actifs de HCRI seraient donc favorisés dans ce contexte. On voit ainsi se dégager les valeurs adaptives de différents allèles d'HCRI, selon les conditions d'inondation affectant les plantes.

\section{Perspectives}

HCRI apparaît comme un déterminant génétique majeur de la réponse adaptative des racines aux inondations [10]. Ce gène régule notamment l'absorption racinaire d'eau en fonction des teneurs combinées en $\mathrm{O}_{2}$ et $\mathrm{K}^{+}$dans le sol. Cette découverte est importante d'un point de vue fondamental car elle révèle des mécanismes moléculaires permettant aux plantes de répondre de manière ciblée à des stress environnementaux combinés. Alors que des études physiologiques antérieures avaient révélé, chez le blé, une interaction entre la nutrition en azote ou phosphate et la tolérance aux inondations [11], le lien entre $\mathrm{O}_{2}$ et $\mathrm{K}^{+}$était resté inconnu jusqu'à présent. Ces résultats ouvrent ainsi de nouvelles perspectives dans le domaine de la sélection et de l'amélioration des plantes cultivées. Les bénéfices éven- tuels du $\mathrm{K}^{+}$dans la réponse des plantes cultivées aux inondations devront être examinés. Il sera par ailleurs intéressant de rechercher des homologues d'HCRI chez ces espèces de plantes cultivées, afin d'introduire les allèles les plus favorables dans de nouvelles variétés. L'utilisation de l'eau et les performances des racines étant des cibles importantes pour l'amélioration des plantes, il faut désormais intégrer, dans les schémas de sélection variétale, leur capacité à adapter la plante à des stress multiples. $\diamond$

Combined adaptation of roots to flooding and soil nutrients:

Role of a MAP3K protein kinase

\section{LIENS D'INTÉRÊT}

Les auteurs déclarent n'avoir aucun lien d'intérêt concernant les données publiées dans cet article.

\section{RéFÉRENCES}

1. Douglas I. Climate change, flooding, and food security in south Asia. Food Sec 2009; $1: 127-36$.

2. Hirabayashi Y, Mahendran R, Koirala S, et al. Global flood risk under climate change. Nat Clim Change 2013 ; 3 : 816-21.

3. Voesenek LA, Bailey-Serres J. Flood adaptive traits and processes: an overview. New Phytol $2015 ; 206$ : 57-73.

4. van Dongen JT, Licausi F. Oxygen sensing and signaling. Annu Rev Plant Biol 2015 ; 66 : 345-67.

5. Thompson CB. Into thin air: How we sense and respond to hypoxia. Cell $2016 ; 167: 9-11$.

6. Gothié $\varepsilon$, Pouysségur J. HIF-1 : régulateur central de I'hypoxie. Med Sci (Paris) $2002 ; 18: 70-8$.

7. Weits DA, Giuntoli B, Kosmacz M, et al. Plant cysteine oxidases control the oxygen-dependent branch of the $\mathrm{N}$-end-rule pathway. Nat Commun $2014 ; 5: 3425$.

8. Shabala S, Shabala L, Barcelo J, Poschenrieder C. Membrane transporters mediating root signalling and adaptive responses to oxygen deprivation and soil flooding. Plant Cell Environ 2014 ; 37 : 2216-33.

9. Maurel C, Boursiac Y, Luu DT, et al. Aquaporins in plants. Physiol Rev 2015 ; 95 : 1321-58.

10. Shahzad Z, Canut M, Tournaire-Roux C, et al. A potassium-dependent oxygen sensing pathway regulates plant root hydraulics. Cell $2016 ; 167$ : 87-98.

11. Guyot C, Prioul JL. Correction par la fertilisation minérale des effets de l'ennoyage sur le blé d'hiver. I. Expérimentation sur sol. Agronomie 1985 ; 5 : 743-50.

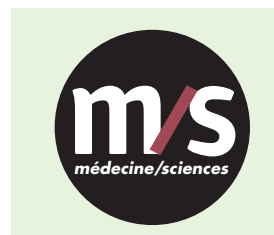

Tarifs d'abonnement $\mathrm{m} / \mathrm{s}-2017$

Abonnez-vous

à médecine/sciences
$>$ Grâce à $m / s$, vivez en direct les progrès des sciences biologiques et médicales

Bulletin d'abonnement page 450 dans ce numéro de $\mathrm{m} / \mathrm{s}$

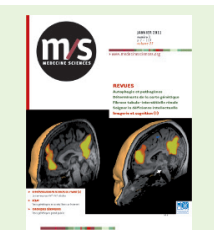

